\title{
Im Sinne des Patientenkomforts
}

Kammerlander, Gerhard ; Geyrhofer, Claudia ; Luch, Gerlinde ; Madar, Felix

Abstract: Vakuumtherapie in Kombination mit aktiver Meersalz-Sauerstofflösung $1 \mathrm{O} 2$

DOI: https://doi.org/10.1007/s00735-015-0459-9

Posted at the Zurich Open Repository and Archive, University of Zurich ZORA URL: https://doi.org/10.5167/uzh-207612

Journal Article

Published Version

Originally published at:

Kammerlander, Gerhard; Geyrhofer, Claudia; Luch, Gerlinde; Madar, Felix (2015). Im Sinne des Patientenkomforts. ProCare, 20(1-2):20.

DOI: https://doi.org/10.1007/s00735-015-0459-9 


\section{Im Sinne des Patientenkomforts}

\section{Vakuumtherapie in Kombination mit aktiver Meersalz-Sauerstofflösung ${ }^{1} \mathrm{O}_{2}$}

Über den Sinn, die Indikation und die Notwendigkeit einer ordnungsgemäßen medizinischen Wundreinigung bzw. auch eines Wund-Débridements liegt eine große Menge von Publikationen vor: Nicht nur aus klassisch chirurgischer Sicht, sondern auch im Zusammenhang mit der Vakuumtherapie (Unterdrucktherapie) und der Reinigung und indikations- und phasengerechten Spülung von Wunden mit Wundspüllösung in der nicht akut-infektiösen Phase bzw. von Antiseptika in der akuten Infektionsphase.

Diese Fragestellungen sollen an dieser Stelle nicht detailliert hinterfragt, sondern vielmehr die Erfahrungsergebnisse in der Anwendung der Vakuumtherapie bei zeitgleicher Anwendung der meersalzbasierten ${ }^{1} \mathrm{O}_{2}$ Wundspüllösung (ActiMaris ${ }^{\circ}$ ) präsentiert werden.

\section{Zielsetzung}

Auf Basis der umfassend dokumentierten Anwendungen können wir aus medizinisch-praktischer Sicht eine Bewertung der klinisch-ambulant durchgeführten lokalen Behandlungen mit der international breit etablierten Vakuumtherapie von Wunden (CNP, NPWT, V.A.C,...) in Kombination mit der benannten Wundspüllösung vornehmen (positive wie negative Erfahrungen).

\footnotetext{
Gerhard Kammerlander, MBA/akad.BO/DGKP/ ZWM $^{\circledast}$, GF - Akademie-ZWM ${ }^{\oplus}$ - KAMMER-LAN DER-WFI Schweiz, GF WKZ ${ }^{\circledR}$-WundKompetenz Zentrum - Linz - A, ZWM ${ }^{\circledR}$-Zertifizierter Wundmanager ${ }^{\circledast}$ auf Basis $\S 64$ GuKG - Embrach - $\mathrm{CH}$, Präsident ARGE ZWM ${ }^{\circledast}$, Lehrbeauftragter Med. Universität Zürich, offizieller TÜV AUSTRIA beauftragter Prüfer für PELLEGRINUS ${ }^{\circledR}$-Wundstandard/AHW ${ }^{\circledR}$-Angewandtes Haut \& Wundmanagement/WZ ${ }^{\circledR}$-WundZentrum/WKZ ${ }^{\circledR}$-WundKompetenzZentrum

2 Claudia Geyrhofer, DGKS/AZWM ${ }^{\circledast}$, PDL WKZ $^{\circledR}$ WundKompetenzZentrum - Linz - A

3 Dr. Gerlinde Luch, FA Orthopädie, Ärztliche Leitung WKZ ${ }^{\circledR}$-WundKompetenzZentrum - Linz - A

4 Felix Madar, DGKP/AZWM ${ }^{\circledast}$, Geschäftsführer der $\mathrm{AHW}^{\circledast}$ basierten WBZ-Wundbehandlungszentren in Wien, Korneuburg und Baden bei Wien
}

\section{Methodik}

Das WKZ-Linz sowie die WBZ-Felix Madar arbeiten seit mehr als sieben bzw. fünf Jahren standardmäßig mit dieser Wundspüllösung. In über 90 Prozent der Anwendungen wird das Produkt für Wundspülungen, Wundreinigungs-Maßnahmen sowie für nasse Umschläge (Nass-Trockenphase) verwendet. Darüber hinaus wurde die Wundspüllösung auch zur Spülung des Vakuumtherapieschaumstoffes (PU-Schaumstoff bzw. der Kerlix Gaze) wiederholt genutzt. Alle Patienten des WKZ-Linz sowie der WBZ-Felix Madar, werden lückenlos schriftlich und bildlich dokumentiert. Auf Basis dieser dokumentierten Daten konnten neben den praktischen Erfahrungen der Therapeuten alle nachfolgend beschriebenen Effekte nachweislich belegt werden.

Die standardmäßige Anwendung der Wundspüllösung hat nicht nur das Ziel die Wunde zu reinigen, sondern soll auch das Wohlbefinden und damit verbunden die erlebte Lebensqualität der Patienten (Schmerzreduktion, Entzündungsreduktion, Reduktion der mikrobiellen Belastung, Reduktion bzw. zeitweise Elimination unangenehmer Gerüche) fördern.

\section{Ergebnisse}

- Die Anzahl der bisher mit der Wundspüllösung behandelten Patienten liegt im $\mathrm{WKZ}^{\circ}$ inklusive dem WBZ bei über 985 Patienten.

- Die gesamte Anwendungshäufigkeit liegt bei insgesamt über 15.700 Anwendungen.

- Davon wurden insgesamt 106 Patienten in Zusammenhang bzw. in Kombination mit der Vakuumtherapie versorgt.

- Aus allergologischer Sicht gab es bisher keine Komplikationen.

- Therapieabbrüche auf Basis von Unverträglichkeiten gab es bisher keine.

- In mehr als 95 Prozent der Fälle wurde die 1,2\%ige Wundspüllösung eingesetzt. In besonders stark fibrinös belegten und stark übelriechenden Wunden wurde die 3\%ige Lösung kurzzeitig eingesetzt.
- Es kam in keinem Fall zu Unverträglichkeiten oder negativen Interaktionen im Zusammenhang mit der Vakuumtherapie.

- Der Großteil der Patienten (über 80\%) empfand die Neutralisation des Wundgeruches und den lokalen beruhigenden Effekt als sehr angenehm.

- Durch das regelmäßige Befragen der Patienten mit einer Lebensqualitätsskala inklusive Schmerzmessung (VAS - 10 Punkte) konnten wir die positiven Veränderungen in diesen Bereichen eindeutig messen.

- Bei weniger als einem Prozent der Patienten kam es zu kurzzeitigem Brennen am Wundgrund, welches sich aber in fast allen Fällen nach wenigen Minuten normalisierte.

\section{Zusammenfassung/Conclusio}

Aus unserer Erfahrung hat sich die Wundspüllösung als Grundmaßnahme in der Nassphase als sehr gut erwiesen.

Auch in Kombination mit der Vakuumtherapie können wir auf Basis der bisherigen Erfahrungen eine klare positive Empfehlung aussprechen.

Negative oder gar gehäuft negative Ereignisse konnten wir bisher nahezu keine feststellen (siehe dazu auch die Auflistungen unter Ergebnisse).

\section{Interessenskonflikt}

Dies ist eine unabhängige prospektive Studie aus dem eigenen Patientenpool der vergangenen sieben $\left(\mathrm{WKZ}^{\circ}\right)$ bzw. vier Jahre (WBZ) im Eigeninteresse der Akademie-ZWM ${ }^{\circ}$, des $W_{K Z}{ }^{\circ}$-Linz, sowie der AHW $^{\circ}$ basierten WBZ-Wund Behandlungszentren-Felix Madar.

Ohne Sponsoring und ohne Auftrag durch Dritte. Es bestehen keine Interessenskonflikte.

\section{Literatur, Publikationen:}

bei den Verfassern

\section{Korrespondenzadresse}

Akademie-ZWM ${ }^{\oplus}$-AG

Gerhard Kammerlander

Taleggstrasse 23, $\mathrm{CH}-8424$ Embrach

kammerlander@wfi.ch

www.wfi.ch 